Original paper

\title{
Histopathologic and electron microscopic investigation of the damage to liver tissue caused by parenteral nutrition combined with starvation in rabbits
}

\author{
Semra Gürünlüoğlu' , Mehmet Gül², Harika Gözükara Bağ \\ 'Department of Pathology, Malatya Education and Research Hospital, Malatya, Turkey \\ 2Department of Histology and Embryology, Faculty of Medicine, Inönü University, Malatya, Turkey \\ ${ }^{3}$ Department of Biostatistics and Medical Informatics, Faculty of Medicine, Inönü University, Malatya, Turkey
}

\begin{abstract}
Aim of the study: To conduct a histopathological examination of the damaging effects of the combination of parenteral nutrition (PN) with starvation on liver tissue using transmission electron and light microscopy.

Material and methods: Four groups ( $n=14$ each) consisting of equal numbers of female and male New Zealand rabbits were formed: a group left completely unfed and receiving full-dose PN (full-dose PN group), a group provided with feed covering half its nutritional needs and receiving half-dose PN (half-dose PN + oral nutrition group), a group provided with feed covering half its nutritional needs (semi-stanvation group), and a group provided with feed covering all its nutritional needs (control group). After 10 days, all rabbits were weighed, anesthetized, and euthanized, and liver tissue samples were collected. Histopathologic examination was performed by a surgical pathologist blinded to the experimental groups. Portal inflammation, ballooning degeneration, apoptosis and fibrosis were evaluated and statistically analyzed.

Results: Severe portal inflammation, moderate portal fibrosis, slight ballooning degeneration, and moderate apoptosis were found in the full-dose PN group. Mild portal inflammation, fibrosis and mild apoptosis were found in the half-dose PN + oral nutrition group. The results of the other two groups were found normal.

Conclusions: Liver damage caused by PN combined with starvation can be devastating. The damage can be minimized by combining PN with enteral nutrition.
\end{abstract}

Key words: liver damage, parenteral nutrition, histopathology.

Address for correspondence:

Semra Gürünlüoğlu, MD, Department of Pathology, Malatya Education and Research Hospital, Malatya, Turkey, e-mail: casemra@yahoo.com

\section{Introduction}

Parenteral nutrition $(\mathrm{PN})$ is the intravenous administration of nutrition when the use of the digestive system is not appropriate [1]. Due to advances in gastrointestinal surgery since the early $19^{\text {th }}$ century, the importance of nutritional support has increased [2]. Nutritional support is one of the factors affecting the success of surgical treatments [2]. In cases in which oral nutrition is not possible, $\mathrm{PN}$ has increased the success of surgical treatments and has been lifesaving [3].

Parenteral nutrition is not an innocuous practice [4-7], and several studies have shown that it can have damaging effects on liver tissue [8-14]. However, none of these studies examined the effects of PN when combined with starvation. Therefore, this study aimed to conduct a histopathologic examination of the damaging effects of the combination of $\mathrm{PN}$ with starvation on liver tissue using transmission electron and light microscopy.

\section{Material and methods}

This study was conducted at the Experimental Animal Production and Research Center of Inonu University. Ethical approval was obtained from the Eth- 
ics Committee for Animal Experimentation of Inonu University (No. 2020/11-1).

\section{Study groups and experimental procedures}

Four groups ( $n=14$ each) consisting of equal numbers of female and male New Zealand rabbits were formed: a group left completely unfed and given fulldose PN (the full-dose PN group), a group provided with feed covering half its nutritional needs and given half-dose PN (the half-dose PN + oral nutrition group), a group provided with feed covering half its nutritional needs (the semi-starvation group), and a group provided with feed covering all its nutritional needs (the control group).

All the animals were weighed at the start of the experiment. The differences in weight between the groups were not significant at the start of the experiment. During the experiment, the rabbits were kept under a 12-hour light and dark cycle with sufficient humidity $(40-50 \%)$ at $18-21^{\circ} \mathrm{C}$. All the rabbits were provided with water ad libitum.

In the rabbits receiving intravenous $\mathrm{PN}$, central catheters were inserted via the internal jugular vein under sterile conditions. During the procedure, $30 \mathrm{mg} / \mathrm{kg}$ ketamine (Alfamine; Ata Fen Veterinary Supplies Inc., İzmir, Turkey) and $5 \mathrm{mg} / \mathrm{kg}$ xylazine (Alfazyne $2 \%$; Alfasan International, Woerden, Netherlands) were administered intramuscularly. A $2 \mathrm{~cm}$ long polyeth- ylene catheter (20 G cutdown catheter; UPS Medical Instruments Co. Ltd., Balgat, Ankara, Turkey) was inserted into the internal jugular vein, with the animal placed in the supine position. The proximal end of the catheter was removed from the back of the vein and fixed through the subcutaneous tunnel.

The parenteral and oral nutrition protocols used in this study were adopted from the work of Gürünlüoğlu et al., with slight modifications [6] (Table 1). The rabbits in the full-dose PN group received a full dose of PN $(230 \mathrm{ml} / \mathrm{kg})$, covering all their daily calorie needs $(204 \mathrm{kcal} / \mathrm{kg})$ every 24 hours (Table 1). The rabbits in the half-dose PN + oral nutrition group were provided with feed pellets covering half of their daily calorie needs $(102 \mathrm{kcal} / \mathrm{kg})$ and a half dose of PN $(115 \mathrm{ml} / \mathrm{kg})$ every 24 hours. The rabbits in the semi-starvation group were fed pellets providing $102 \mathrm{kcal} / \mathrm{kg}$ per day. The rabbits in the control group were provided with pellets covering all their daily calorie needs (204 kcal $/ \mathrm{kg}$ ).

The PN formula contained $10 \%$ amino acids (w/v) (Primene; E. Baxter, Istanbul, Turkey), trace elements (Addamel N; Fresenius Kabi, Uppsala, Sweden), 20\% (w/v) lipids (Lipofundin MCT/LCT 20\%; B. Braun, Melsungen, Germany), and 30\% glucose (dextrose 30\%; Polyflex; Polypharma Pharmaceuticals S.A., Tekirdağ, Turkey) (Table 1). The rabbit pellets contained $18.75 \%$ protein, $6.38 \%$ cellulose, $2.28 \%$ fat, $6.2 \%$ ash, $2.45 \%$ minerals, $0.98 \%$ lysine, $0.36 \%$ methionine,

Table 1. Parenteral nutrition (PN) solution formula (total liquid volume $230 \mathrm{ml} / \mathrm{kg} / \mathrm{d}$ and total calories $204 \mathrm{kcal} / \mathrm{kg} / \mathrm{d}$ )

\begin{tabular}{|c|c|c|c|c|c|c|}
\hline \multirow[t]{2}{*}{ Ingredient } & \multicolumn{2}{|c|}{ Volume (ml) } & \multicolumn{2}{|c|}{ Calories (kcal) } & \multicolumn{2}{|c|}{ Calories percentage $(\%)$} \\
\hline & Full-dose PN & $\begin{array}{l}\text { Half dose PN } \\
\text { + oral nutrition }\end{array}$ & Full-dose PN & $\begin{array}{l}\text { Half dose PN } \\
\text { + oral nutrition }\end{array}$ & Full-dose PN & $\begin{array}{l}\text { Half dose PN } \\
+ \text { oral nutrition }\end{array}$ \\
\hline $20 \%$ medium long-chain fat emulsion (1) & 38 & 19 & 72.2 & 36.1 & 35 & 17.4 \\
\hline $10 \%$ compound amino acids & 88 & 44 & 34.8 & 17.4 & 17 & 8.9 \\
\hline $30 \%$ glucose & 80 & 40 & 97 & 48.5 & 48 & 24 \\
\hline $3 \%$ sodium chloride & 13 & 6.5 & & & & \\
\hline $10 \%$ potassium chloride & 3 & 1 & & & & \\
\hline $10 \%$ calcium glukonate & 3 & 1 & & & & \\
\hline Water-soluble vitamins (2) & 1/2 ampoule & $1 / 4$ ampoule & & & & \\
\hline Fat-soluble vitamins (3) & 1/2 ampoule & 1/4 ampoule & & & & \\
\hline Trace elements (4) & 1 & 0.5 & & & & \\
\hline Total & 230 & 115 & 204 & 102 & & \\
\hline
\end{tabular}

(1) medium/long-chain fat emulsion (250 ml) composition: soybean oil $25 \mathrm{~g}$, medium chain triglycerides $25 \mathrm{~g}$ and lecithin $3 \mathrm{~g}$, linoleic acid $13 \mathrm{~g}$, $\alpha$-linolenic acid $1.5 \mathrm{~g}$, $3 \mathrm{~g}$ egg phospholipids.

(2) Water-soluble vitamins composition: vitamin $B_{1} 0.5 \mathrm{mg}$, vitamin $B_{2} 0.7 \mathrm{mg}$, nicotinamide $8 \mathrm{mg}$, vitamin $B_{6} 0.2 \mathrm{mg}$, pantothenic acid $3 \mathrm{mg}$, vitamin C $20 \mathrm{mg}$, biotin $12 \mu \mathrm{g}$, folic acid $80 \mu \mathrm{g}$, vitamin B $100 \mu \mathrm{g}$.

(3) Fat soluble vitamins composition: vitamin A $50 \mu \mathrm{g}$ (165 IU), vitamin D $20.25 \mu \mathrm{g}$ (10 IU), vitamin E $0.455 \mu \mathrm{g}$ (0.5 IU) and vitamin K $7.5 \mu \mathrm{g}$.

(4) Trace elements: Chromic chloride $6 \mathrm{H}_{2} 05.33 \mu \mathrm{g} / \mathrm{ml}$, Copper chloride $2 \mathrm{H}_{2} 00.34 \mathrm{mg} / \mathrm{ml}$, Ferric chloride $6 \mathrm{H}_{2} \mathrm{O} 0.54 \mathrm{mg} / \mathrm{ml}$, Manganese chloride $4 \mathrm{H}, 0 \mathrm{g9} .0 \mu \mathrm{\mu g} / \mathrm{ml}$, Potassium iodide $16.6 \mu \mathrm{g} / \mathrm{ml}$, Sodium fluoride $0.21 \mathrm{mg} / \mathrm{ml}$, Sodium molybdate $2 \mathrm{H}_{2} 04.85 \mu \mathrm{g} / \mathrm{ml}$, Sodium selenite anhydrous $6.90 \mu \mathrm{g} / \mathrm{ml}$, Zinc chloride $1.36 \mathrm{mg} / \mathrm{ml}$. 
and $12 \%$ starch $(240 \mathrm{kcal} / 100 \mathrm{~g}$; TV-01 rabbit pellet feed; DSA Agrifood Products Inc., Kırıkkale, Turkey).

After 10 days, all the rabbits were weighed, anesthetized, and euthanized, and liver tissue samples were collected.

\section{Histopathologic methods}

\section{Collection and preparation of liver tissue samples}

After the rabbits were euthanized, the abdomen of each one was incised, and the liver was removed in one piece and washed with serum saline. A 2-3 cm wide and $1 \mathrm{~cm}$ thick tissue sample about $1 \mathrm{~cm}$ away from the liver capsule near the hilar area was removed and kept in 10\% formalin for 24 hours. A $2 \mathrm{~mm}$ wide and $1 \mathrm{~mm}$ thick piece of tissue was removed from the same area, washed with serum saline, and kept in $2.5 \%$ glutaraldehyde for 24 hours for electron microscopic examination.

After 24 hours, the samples kept in 10\% formalin were sliced into pieces of 3-4 mm thickness. One piece from each sample was processed following standard procedures and buried in a paraffin block. Then sections of 3-5 $\mu \mathrm{m}$ thickness were taken from each paraffin block. The sections were stained with routine hematoxylin and eosin ( $\mathrm{H} \& \mathrm{E})$, routine Masson's trichrome (MT) procedures, and anti-caspase- 3 antibodies for immunohistochemical analysis.

\section{Immunohistochemical methods}

The tissue sections were deparaffinized and placed on adhesive slides. The slides were then placed in citrate buffer ( $\mathrm{pH}$ 7.6; Thermo Scientific, Fremont, CA, USA), and antigen retrieval was performed in a pressure cooker (Retriever 2100; Aptum, Southampton, UK) at $121^{\circ} \mathrm{C}$ for 15 minutes. The sections were then left to cool, the citrate buffer was removed, and phosphate-buffered saline was added. The slides were washed with phosphate-buffered saline at every stage, except during protein blocking and primary antibody application.

The following steps were carried out at room temperature. A horseradish peroxidase kit (Thermo Scientific) was used for immunostaining according to the manufacturer's protocol. First, 3\% hydrogen peroxide was dripped for 10 minutes. Second, a protein V-blocking solution was dripped for five minutes, followed by incubation with caspase-3 primary antibody (anticaspase-3 antibody [ABM1C12] ab208161; Abcam, Cambridge, UK; dilution ratio $1: 300)$ at room tem- perature for one hour. Third, the glass slides were incubated in a biotinylated goat antipolyvalent secondary antibody for 10 minutes. Streptavidin peroxidase was dripped and incubated for 10 minutes. Aminoethyl carbazole chromogen (Thermo Scientific) was dripped and incubated for 15 minutes. Finally, the slides were placed in distilled water, counterstained with Mayer's hematoxylin, and mounted.

\section{Scoring and histopathologic evaluation of stained liver tissue sections}

Histopathologic evaluation was performed by a surgical pathologist who was blinded to the experimental groups. We evaluated portal inflammation, ballooning degeneration in the $\mathrm{H} \& \mathrm{E}$-stained sections, and fibrosis in the MT-stained sections. In this study, we based the scoring system on the studies of Curran et al. [15] and Kohl et al. [16]. We adapted the scoring systems in these two studies with a few modifications. The scoring system we used is shown in Table 2.

The presence of apoptotic cells was evaluated in the H\&E-stained sections. Apoptotic cells were characterized as round to oval-shaped cells with a dark eosinophilic/ dense cytoplasm and purple dense pyknotic/fragmented nuclei or fragmented apoptotic cells forming apoptotic bodies. To avoid mistaking single-cell necrosis for apoptosis, cells surrounded by inflammatory cells were not taken into consideration, even if they met the morphological criteria [17]. Eight 400× magnification areas were evaluated for each sample [17]. Apoptotic cells and the total hepatocytes (approximately 3,500 cells) in those areas were counted for each rabbit, and the ratio of apoptotic cells to total hepatocytes was calculated as an apoptosis score $[14,17]$.

Caspase-3-stained sections were evaluated by counting the positively stained cells in the $400 \times$ magnification areas. The ratio of stained cells to total cells was calculated for each rabbit $[14,17]$.

\section{Transmission electron microscopic examination}

For transmission electron microscopic examination, liver tissue samples were fixed in $2.5 \%$ glutaraldehyde buffered with $0.2 \mathrm{M} \mathrm{NaH}_{2} \mathrm{PO}_{4}+\mathrm{NaHPO}_{4}(\mathrm{pH}$ 7.4) and postfixed in $1 \% \mathrm{OsO}_{4}$. After dehydration in acetone, they were embedded in epoxy resin (Araldite CY212). Thin sections $(80 \mathrm{~nm})$ were cut from Araldite blocks with an ultramicrotome and contrasted with uranyl acetate and lead citrate. They were then examined under a transmission electron microscope (Libra 120; Carl Zeiss NTS, Oberkochen, Germany) and photographed. Intracytoplasmic edema, organelle degeneration, and 
Table 2. Histopathological scoring system

\begin{tabular}{lccc}
\hline Score & Ballooning degeneration & Inflammation & Fibrosis \\
\hline 0 & None & None & None \\
\hline 1 & $\begin{array}{c}\text { Mild, degenerated cells covering }<25 \% \\
\text { of the porto-central distance }\end{array}$ & $\begin{array}{c}\text { Mild, leukocytes covering }<25 \% \\
\text { of the portal area }\end{array}$ & $\begin{array}{c}\text { Mild increase in connective tissue, } \\
\text { limited to the portal area }\end{array}$ \\
\hline 2 & $\begin{array}{c}\text { Moderate, degenerated cells covering } 25-50 \% \\
\text { of the porto-central distance }\end{array}$ & $\begin{array}{c}\text { Moderate, leukocytes covering } 25-50 \% \\
\text { of the portal area }\end{array}$ & Moderate, fibrosis beyond the portal area \\
\hline 3 & $\begin{array}{c}\text { Severe, degenerated cells covering }>50 \% \\
\text { of the porto-central distance }\end{array}$ & Severe, leukocytes beyond the portal area & Severe fibrosis with porto-portal bridging \\
\hline
\end{tabular}

apoptotic-looking nuclei with reduced diameters in heterochromatin structures were investigated.

\section{Statistical analysis}

Values were expressed as medians and ranges. The Kruskal-Wallis test was used for comparisons between the groups. Pairwise comparisons were then performed using the Conover method. In all the tests, a two-sided value of $p<0.05$ was considered statistically significant.

\section{Results}

\section{Weight changes}

Table 3 shows the results of statistical analysis of the weight changes in the four groups. The differences in weight change between the two PN groups and the control group were not statistically significant. However, the difference in weight change between the semi-starvation group and the other groups was statistically significant.

\section{Histopathologic results}

The results of the histopathological examination are displayed in Table 4. Moderate portal fibrosis was found in the full-dose PN group, while mild fibrosis was observed in the half-dose PN + oral nutrition group. Conversely, no fibrosis was detected in the semi-starvation and control groups (Fig. 1A, B). Severe portal inflammation was found in the full-dose PN group, while mild inflammation was observed in the half-dose $\mathrm{PN}+$ oral nutrition group. In contrast, no inflammation was detected in the semi-starvation and control groups (Fig. 1C, D). Slight ballooning degeneration was observed in the full-dose PN group. Conversely, no ballooning degeneration was detected in the other three groups (Fig. 2A). Moderate apoptosis was found in the full-dose PN group, while mild apoptosis was detected in the half-dose PN + oral nutrition group. Minimal apoptosis was found in the semi-starvation and control groups (Figs. 2B and 3A). Moderate caspase-3 expression was detected in the full-dose PN group, while mild expression was found in the half-dose $\mathrm{PN}+$ oral nutrition group.

Table 3. Statistical analysis of the change in weight of rabbits in study groups during the study period

\begin{tabular}{lccc}
\hline Groups & Weight before experiment $(\mathbf{g})$ & Weight after experiment $\mathbf{( g )}$ & $\boldsymbol{P}$-value \\
\hline Control $(n=14)$ & $3390 \pm 107$ & $3392 \pm 119$ & 0.931 \\
\hline Semi-starvation $(n=14)$ & $3349 \pm 155$ & $3183 \pm 124$ & $<0.001$ \\
\hline Full-dose PN $(n=14)$ & $3408 \pm 106$ & $3392 \pm 85$ & 0.226 \\
\hline Half-dose PN + oral nutrition $(n=14)$ & $3374 \pm 108$ & $3367 \pm 137$ & 0.631 \\
\hline
\end{tabular}

$P N-$ parenteral nutrition

Table 4. Histopathological and immunohistochemical evaluation results

\begin{tabular}{lcccc}
\hline & Control $(\boldsymbol{n}=\mathbf{1 4})$ & Semi-starvation $(\boldsymbol{n}=\mathbf{1 4})$ & Half-dose PN + oral nutrition $(\boldsymbol{n}=14)$ & Full-dose PN $(\boldsymbol{n}=14)$ \\
\hline Portal fibrosis & $0(0-0)^{\mathrm{a}}$ & $0(0-0)^{\mathrm{a}}$ & $1(1-2)^{\mathrm{b}}$ & $2(1-3)^{\mathrm{c}}$ \\
\hline Apoptosis & $0.01(0.01-0.02)^{\mathrm{a}}$ & $0.01(0.01-0.02)^{\mathrm{a}}$ & $0.05(0.04-0.06)^{\mathrm{b}}$ & $0.23(0.11-0.43)^{\mathrm{c}}$ \\
\hline Portal inflammation & $0(0-1)^{\mathrm{a}}$ & $0(0-1)^{\mathrm{a}}$ & $1(1-2)^{\mathrm{b}}$ & $3(1-3)^{\mathrm{c}}$ \\
\hline Balloon degeneration & $0(0-0)^{\mathrm{a}}$ & $0(0-0)^{\mathrm{a}}$ & $0(0-1)^{\mathrm{a}}$ & $1(1-2)^{\mathrm{b}}$ \\
\hline Caspase-3 expression & $0.01(0.01-0.03)^{\mathrm{a}}$ & $0.01(0.01-0.03)^{\mathrm{a}}$ & $0.04(0.03-0.06)^{\mathrm{b}}$ & $0.48(0.25-0.76)^{\mathrm{c}}$ \\
\hline P-value & $<0.001$ & $<0.001$ & $<0.001$ & $<0.001$
\end{tabular}

*The difference between the groups with different superscript letters are statistically significant.

$P N$ - parenteral nutrition 

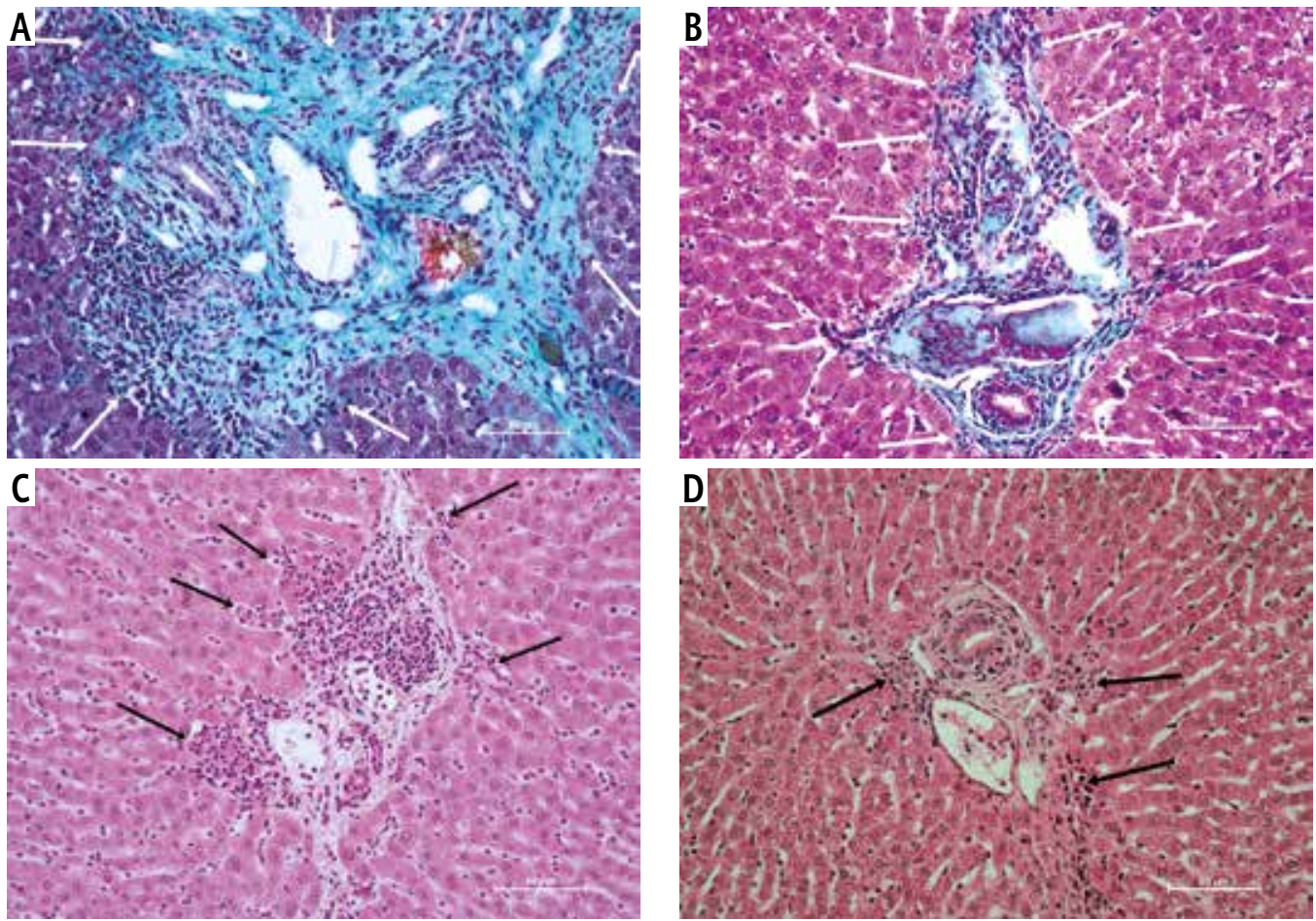

Fig. 1. Light microscope liver tissue images of the parenteral nutrition (PN) groups. A) Fibrosis beyond the portal area in the full-dose PN group (white arrows) (Masson's trichrome, 200x magnification). B) The portal area of the half-dose PN + oral nutrition group shows a mild degree of fibrosis confined to the portal area (white arrows) (Masson's trichrome, 200x magnification). C) Severe degree of inflammation in the portal and periportal area of a subject from the full-dose PN group (black arrows) (hematoxylin and eosin, 200x magnification). D) Mild degree of portal inflammation in a subject from the half-dose PN + oral nutrition group (black arrows) (hematoxylin and eosin, 200x magnification)
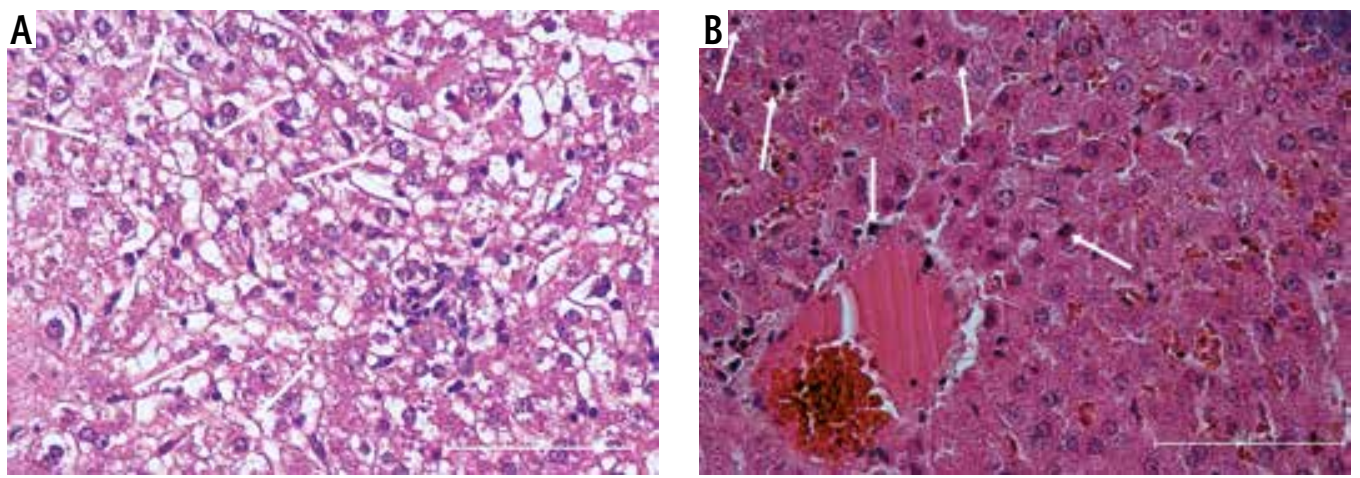

Fig. 2. A) In this picture, which belongs to the full-dose parenteral nutrition group, some hepatocytes with balloon degeneration are shown with white arrows (hematoxylin and eosin, 400x magnification). B) Some apoptotic cells are indicated with white arrows here. The picture belongs to a subject from the full-dose parenteral nutrition group (hematoxylin and eosin, 400x magnification)

Minimal expression was found in the semi-starvation and control groups (Figs. 3B and 4A-C). All differences were statistically significant $(p<0.001)$.

\section{Transmission electron microscopic findings}

The ultrastructural appearance of liver sections of the half-dose PN + oral nutrition, semi-starvation, and control groups was normal (Fig. 5A). In contrast, the liver sections of the full-dose PN group exhibited profound ultrastructural changes in hepatocytes. Many lipid vacuoles of different diameters and variably sized membrane-bound vacu- oles containing electron-dense material, intracytoplasmic edema, and accumulated electron-dense glycogen granules were observed in the hepatocyte cytoplasm (Fig. 5B, C).

\section{Evaluation of results}

The histopathologic results suggest that $\mathrm{PN}$ combined with starvation could cause moderate portal fibrosis and apoptosis, severe portal inflammation, and mild ballooning degeneration. When $\mathrm{PN}$ was combined with oral nutrition, portal inflammation, portal fibrosis, apoptosis, and caspase-3 expression were milder. Although liver 

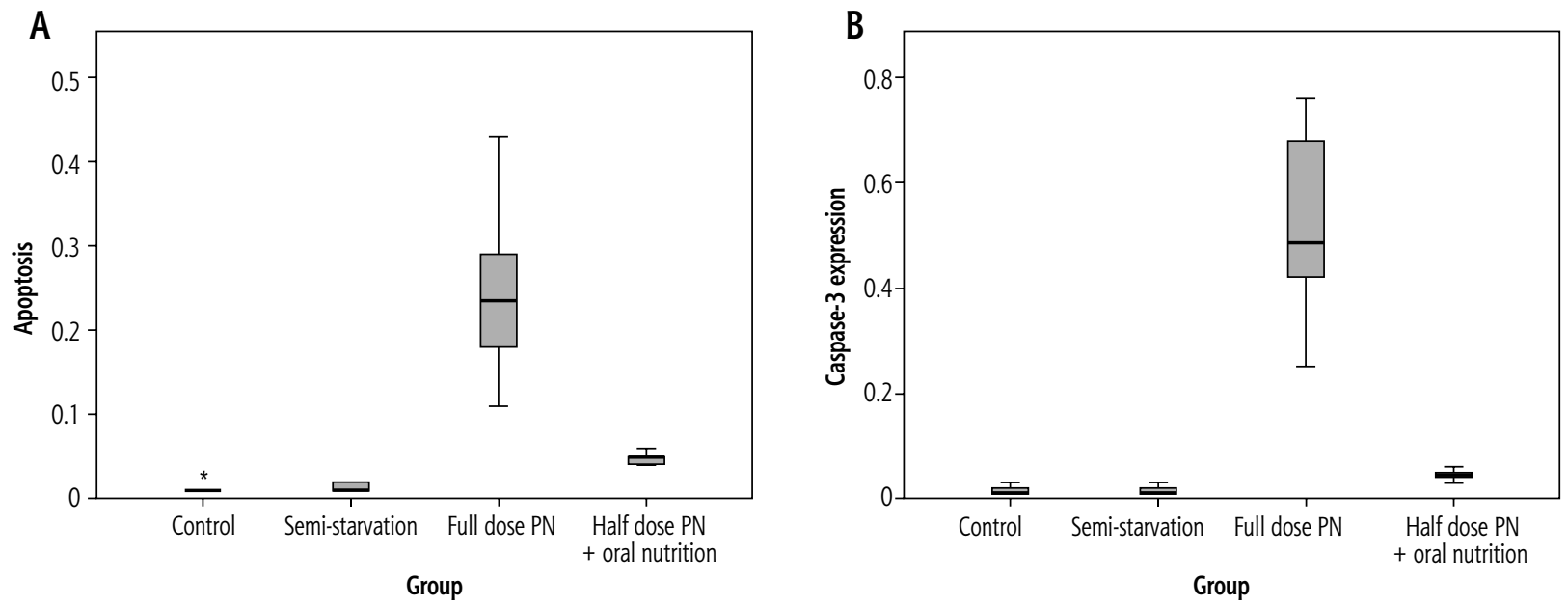

Fig. 3. Liver tissue apoptosis and caspase-3 expression evaluation results of the four groups. A) Apoptosis findings evaluation results. B) Caspase-3 expression evaluation results
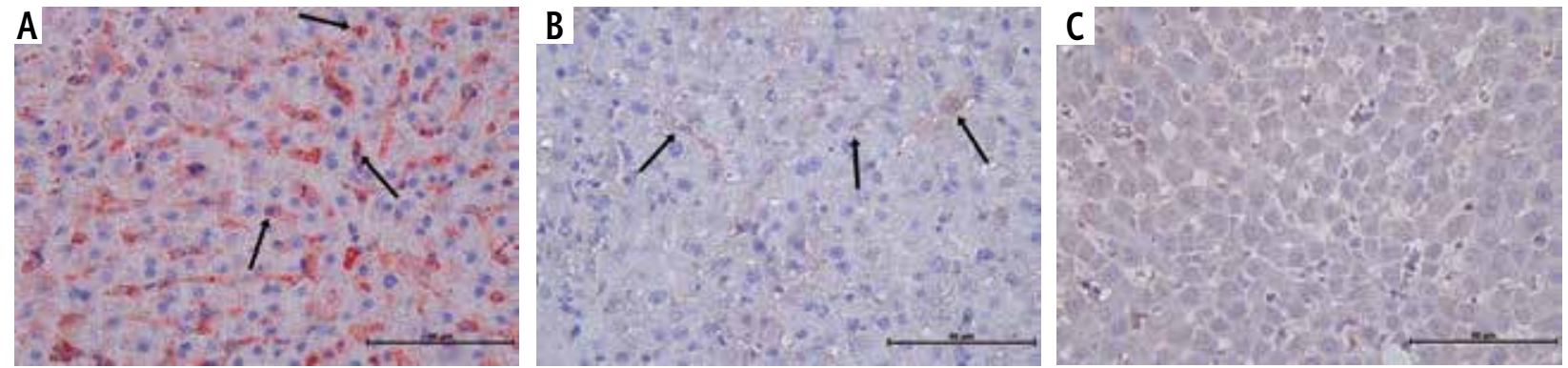

Fig. 4. Light microscope images of liver tissue caspase-3 expression. A) This picture shows severe caspase-3 expression (black arrows) in the full-dose PN group. B) This picture shows mild caspase-3 expression (black arrows) in the half-dose PN + oral nutrition group. C) Control group image
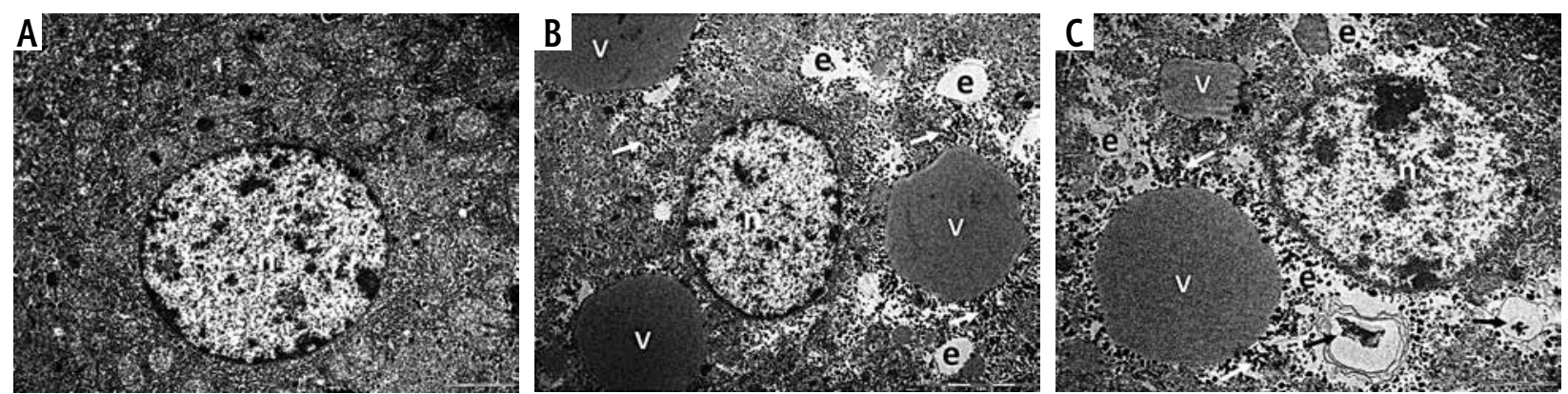

Fig. 5. A) Half-dose parenteral nutrition (PN) + oral nutrition group: normal ultrastructural appearance of hepatocyte, hepatocyte nuclei (n). Uranyl acetate-lead citrate, transmission electron microscope (TEM), scale bar $=2 \mu \mathrm{m}$, original 6300x magnification. B) Full-dose PN group: hepatocyte nuclei (n) intracytoplasmic lipid vacuole (v), intracytoplasmic edema (e), glycogen granules (arrow) in hepatocytes. Uranyl acetate-lead citrate, TEM, scale bar $=5 \mu \mathrm{m}$, original 4000x magnification. C) Full-dose PN group: hepatocyte nuclei (n), intracytoplasmic lipid vacuole (v), intracytoplasmic edema (e), glycogen granules (white arrow), membrane-bound vacuole containing electron-dense material in hepatocytes (black arrow). Uranyl acetate-lead citrate, TEM, scale bar $=5 \mu \mathrm{m}$, original 6000x magnification

damage still occurred, a devastating effect was avoided. Our findings also showed that hunger itself, which resulted in slight weight loss, does not cause damage to the liver.

\section{Discussion}

Many studies have investigated the negative effects of PN on the liver [8-15]. These studies have mainly fo- cused on the components of PN formulas [8-15]. Our study is the first to investigate how the damaging effect of PN on the liver can be minimized by combining it with oral feeding.

The development of steatosis in liver tissue is one of the important negative effects of PN [1]. A study on newborn piglets reported that $\mathrm{PN}$ caused steatosis in liver tissue [8]. The authors found that the molecular and 
morphological pathogenesis of PN-related liver damage is associated with apoptosis mediated by the mitochondrial and Fas pathways [8]. In our study, we found that PN caused moderate apoptosis in liver tissue in the case of total starvation, but the effect was quite mild when $\mathrm{PN}$ was combined with enteral nutrition.

An experimental study investigating the relationship between liver damage and oxidative stress caused by PN found portal inflammation, proliferation of bile ducts, increased bile pigments in hepatocytes, and diffuse steatosis in the entire liver [14]. The authors suggested that the severity of the damage depends on the duration of $\mathrm{PN}$ administration [14].

Some studies have reported that the liver damage caused by PN is associated with the lipids in its formula [18-20]. For example, Tazuke et al. [18] found that the damage to the liver caused by $\mathrm{PN}$ is due to the lipids in its composition, which causes apoptosis. Similarly, La Scala et al. [19] found that cholestasis developing during $\mathrm{PN}$ is associated with the lipids contained in the formula. Conversely, the authors suggested that hepatocellular damage was not associated with lipids [19]. Nakagawa et al. found that medium-chain triglycerides (MCT) in PN formulas caused steatosis in the liver due to their disruptive effect on nitrogen balance and albumin levels, whereas PN formulas containing long-chain triglycerides caused milder fatty changes in the liver [20].

Various lipid emulsions are used in PN compositions [21]. Intralipid, made from soybean oil, contains a-tocopherol, oleic acid, linoleic acid, palmitic acid, a-linolenic acid, stearic acid, and the highest phytosterol concentration of all lipid emulsions [21, 22]. SMOFlipid $20 \%$ is a mixture of soybean oil, MCT, fish oil, and olive oil and contains $\alpha$-tocopherol. It consists of eicosapentaenoic acid, $\alpha$-linolenic acid, linoleic acid, palmitic acid, oleic acid, docosahexaenoic acid, arachidonic acid, and stearic acid and contains less phytosterol than other lipid emulsions [21, 22]. Lipofundin $20 \%$ is produced from soybean oil and contains MCT and moderate amounts of phytosterol. It consists of palmitic acid, arachidonic acid, oleic acid, $\alpha$-linolenic acid, stearic acid, and linoleic acid. Omegaven is produced from fish oil. It consists of egg phospholipids, eicosapentaenoic acid, linoleic acid, docosahexaenoic acid, $\alpha$-linolenic acid, and glycerol and contains no phytosterol [23].

Cholestasis during $\mathrm{PN}$ has been associated with the phytosterol content of the lipid emulsions [21]. Phytosterol omega- 6 contains polyunsaturated fatty acids, which can cause cholestasis [21]. Omega-3 fatty acids, on the other hand, have been found to exert a hepatoprotective effect [21]. Lipofundin has been associ- ated with cholestasis due to its omega- 6 content [21], whereas SMOFlipid and Omegaven have been suggested to exert a hepatoprotective effect [21,23]. However, because SMOFlipid is poor in lipids and Omegaven does not contain phytosterol, which is vital for the development of the central nervous system and retina, their long-term use is not recommended in preterm infants [24].

In this study, we used Lipofundin 20\%. However, although the combination of $\mathrm{PN}$ and starvation caused moderate liver disease, the combination of $\mathrm{PN}$ with enteral nutrition caused only mild damage. We believe that when enteral nutrition is possible, it can reduce the damaging effects of $\mathrm{PN}$ on the liver to some extent.

Amino acid solutions contained in $\mathrm{PN}$ formulas can significantly contribute to the damage caused to the liver. Bellini et al. reported that the composition of amino acid solutions contained in PN formulas plays an important role and that the presence of methyl donor amino acids could mitigate the cholestatic effect of $\mathrm{PN}$ on the liver [25]. Hat et al. suggested that amino acid solutions in appropriate concentrations could be effective in preventing liver damage [26]. The PN formula used in our study contained $10 \%$ amino acid solution.

$\mathrm{PN}$ is a nutritional technique in which fluids and nutrients needed by the body are given intravenously. We speculate that in the event of a complete disabling of the gastrointestinal tract, hormonal regulatory mechanisms are completely disabled, thus not ensuring proper distribution and proper use of all components of PN at the cellular level in the body. This may have set the stage for some negative conditions in the body, such as hyperglycemia and oxidative stress. Under normal circumstances, we believe that these hormones are released from the digestive tract in the case of nutrients entering [6]. We think that the severity of the liver damage, which is related to PN combined with hunger, may be associated with a lack of nutrient inflow into the digestive tract. We speculate that these negative effects of PN combined with hunger on the liver can be minimized by adding oral nutrition to PN.

\section{Conclusions}

Parenteral nutrition may cause damage to the liver, which can be devastating when combined with starvation. However, the damage can be minimized by combining PN with enteral nutrition.

\section{Acknowledgments}

We would like to thank Kubilay Gürünlüoğlu for his valuable contribution to this study. 


\section{Disclosure}

The authors declare no conflict of interest.

\section{References}

1. Teitelbaum DH, Coran AG. Nutritional support. In: Grosfeld JL, O’Neill JA, Fonkalsrud EW, Coran AG (Eds.). Textbook of Pediatric Surgery. 6th ed. Vol. 1, Chapter 12. Philadelphia 2006; 194-220.

2. Dudrick SJ. Early developments and clinical applications of total parenteral nutrition. J Parenter Enteral Nutr 2003; 27: 291-299.

3. Ward N. Nutrition support to patients undergoing gastrointestinal surgery. Nutr J 2003; 2: 1-5.

4. Demircan M, Gürünlüoğlu K, Karaman A, Mizrak B. Damaging effects of total parenteral nutrition Formula on vascular endothelium. J Pediatr Gastroenterol Nutr 2015; 61: 464-468.

5. Buchman AL, Neely M, Grossie VB Jr, et al. Organ heavy-metal accumulation during parenteral nutrition is associated with pathologic abnormalities in rats. Nutrition 2001; 17: 600-606.

6. Gürünlüoğlu K, Gül M, Koçbıyık A, et al. Investigation of the cardiotoxic effects of parenteral nutrition in rabbits. J Pediatr Surg 2020; 55: 465-474.

7. Wildhaber BE, Lynn KN, Yang H, Teitelbaum DH. Total parenteral nutrition-induced apoptosis in mouse intestinal epithelium: regulation by the Bcl-2 protein family. Pediatr Surg Int 2002; 18: 570-575.

8. Wang H, Khaoustov IV, Krishnan B, et al. Total parenteral nutrition induces liver steatosis and apoptosis in neonatal piglets. J Nutr 2006; 136: 2547-2552.

9. Demircan M, Ergün O, Coker C, et al. Aluminium in total parenteral nutrition solutions produces portal inflammation in rats. J Pediatr Gastroenterol Nutr 1998; 26: 274-278.

10. Zhu X, Zhang X, Yu L, et al. Hepatic overexpression of GRP94 in a rabbit model of parenteral nutrition-associated liver disease. Gastroenterol Res Pract 2015; 2015: 1-8.

11. Teitelbaum DH. Parenteral nutrition-associated cholestazis. Curr Opin Pediatr 1997; 9: 270-275.

12. Matilla B, Ortiz J, Gonzalez P, et al. Effects of parenteral nutrition supplemented with glutamine or glutamine dipeptides on liver antioxidant and detoxication systems in rats. Nutrition 2000; 16: 125-128.

13. Chesse P, Lavoie JC, Rouleau T, et al. Photooxidation of parenteral multivitamins induces hepatic steatosis in a neonatal guinea pig model of intravenous nutrition. Pediatr Res 2002; 52: 958-963.

14. Cai W, Wu J, Hong L, et al. Oxidative injury and hepatocyte apoptosis in total parenteral nutrition-associated liver dysfunction. J Ped Surg 2006; 41: 1663-1668.

15. Curran TJ, Uzoaru I, Das JB, et al. The effect of cholecystokinin-octapeptide on the hepatobiliary dysfunction caused by total parenteral nutrition. J Pediatr Surg 1995; 30: 242-246.

16. Kohl M, Wedel T, Entenmann A, et al. Influence of different intravenous lipid emulsions on hepatobiliary dysfunction in a rabbit model. J Pediatr Gastroenterol Nutr 2007; 44: 237-244.

17. Archana M, Bastian, Yogesh TL, Kumaraswamy KL. Various methods avalaible for detection of apoptotic cells - a review. Indian J Cancer 2013; 50: 274-283.

18. Tazuke Y, Drongowski RA, Btaiche I, et al. Effects of lipid administration on liver apoptotic signals in a Mouse model of total parenteral nutrition. Pediatr Surg Int 2004; 20: 224-228.
19. La Scala GC, Le Coultre C, Roche BG, et al. The addition of lipids incereses the total parenteral nutrition-associated cholestasis in the rat. Eur J Pediatr Surg 1993; 3: 224-227.

20. Nakagawa M, Hiramatsu Y, Mitsuyoshi K, et al. Effect of various lipid emulsions on total parenteral nutrition-induced hepatosteatosis in rats. JPEN J Parenter Enteral Nutr 1991; 15: 137-143.

21. Raman M, Almutairdi A, Mulesa L, et al. Parenteral nutrition and lipids. Nutrients 2017; 9: 2-11.

22. Pichler J, Simchowitz V, Macdonald S, Hill S. Comparison of liver function with two new/mixed intravenous lipid emulsions in children with intestinal failure. Eur J Clin Nutr 2014; 68: 11611167.

23. Park KT, Nespor C, Kerner J Jr. The use of omegaven in treating parenteral nutrition- associated liver disease. J Perinatol 2011; 31: 57-60.

24. Riskin A, Hartman C, Shamir R. Parenteral nutrition in very low birth weight preterm infants. Isr Med Assoc J 2015; 17: 310-315.

25. Bellini DC, Fournier LA, Lepage G, et al. Total parenteral nutrition-associated cholestazis in rats: comparison of different aminoacid mixtures. JPEN J Parenter Enteral Nutr 1987; 11: 67-73.

26. Hata S, Nezu R, Kubota A, et al. Effect of amino acids in total parenteral nutrition on cholestasis in newborn rabbits. J Pediatr Surg 1994; 29: 892-895. 